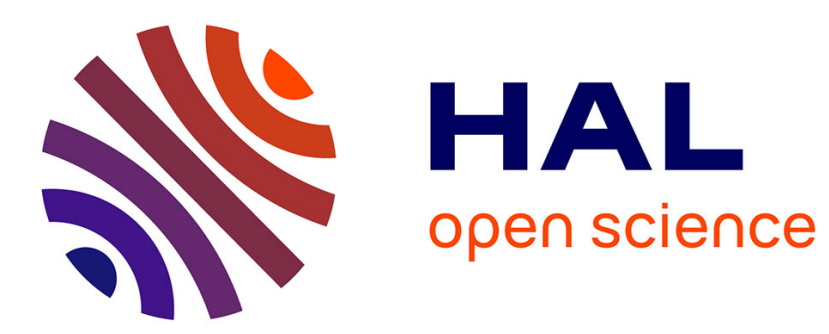

\title{
Atomic plasma excitations in the field of a soft x-ray laser \\ M Richter
}

\section{To cite this version:}

M Richter. Atomic plasma excitations in the field of a soft x-ray laser. Journal of Physics B: Atomic, Molecular and Optical Physics, 2011, 44 (7), pp.75601. 10.1088/0953-4075/44/7/075601. hal-00608729

\section{HAL Id: hal-00608729 \\ https://hal.science/hal-00608729}

Submitted on 14 Jul 2011

HAL is a multi-disciplinary open access archive for the deposit and dissemination of scientific research documents, whether they are published or not. The documents may come from teaching and research institutions in France or abroad, or from public or private research centers.
L'archive ouverte pluridisciplinaire HAL, est destinée au dépôt et à la diffusion de documents scientifiques de niveau recherche, publiés ou non, émanant des établissements d'enseignement et de recherche français ou étrangers, des laboratoires publics ou privés. 


\title{
Atomic plasma excitations in the field of a soft $x$-ray laser
}

\author{
M Richter \\ Physikalisch-Technische Bundesanstalt, Abbestraße 2-12, 10587 Berlin, Germany \\ E-mail: mathias.richter@ptb.de
}

\begin{abstract}
The interaction of atoms with short-wavelength radiation at ultra-high intensities is described by plasma excitation. In contrast to former works on optical radiation and ponderomotive motion of quasi-free electrons, the excitation of correlated and bound electrons is considered here. The ponderomotive motion of a free electron is included as a special case. Values for the energy transfer from the radiation field to an atom are obtained in fair agreement with the unexpectedly high charge states of xenon recently observed at the soft x-ray free electron laser FLASH.
\end{abstract}

PACS numbers: $32.80 . \mathrm{Aa}, 32.80 . \mathrm{Rm}, 41.60 . \mathrm{Cr}, 42.50 . \mathrm{Hz}$ 


\section{Introduction}

With the recent set-up of the first x-ray free-electron laser (FEL) facilities in Europe [1], Japan [2], and the United States [3], a long-cherished vision of the science community has come true. The highly intense and ultra-short soft and hard x-ray laser pulses allow time-resolved imaging of advanced materials with femtosecond resolution for many applications [4-7]. It concerns, for example, the investigation of fast chemical reactions on surfaces or within biological systems by means of microscopic snapshots. The first experiments in microbeams have, however, demonstrated that the mechanisms of photon-matter interaction with high-intensity x-rays may be affected by nonlinear processes which elude a description by the current theoretical models developed for high-intensity radiation in the optical regime $[8,9]$. The latter concerns, in particular, the semi-classical theories based on the ponderomotive motion of individual quasi-free Rydberg electrons driven by the oscillating field of the electromagnetic wave [10-12].

In the present theoretical work, the specific aspects of $\mathrm{x}$-rays, namely excitations of correlated and bound inner-shell electrons are considered. It was initiated by the surprising results of the Xe photoionization experiments performed at the free electron laser FLASH [8, 9]. In the so-called extreme ultra-violet (EUV) spectral sub-range at around $90 \mathrm{eV}$ photon energy and at irradiance levels in the order of $10^{16} \mathrm{~W} \mathrm{~cm}^{-2}$, charge states up to $\mathrm{Xe}^{21+}$ were observed whose generation, starting from neutral Xe atoms, requires a total energy of more than 5,000 eV. Thus, almost $60 \mathrm{EUV}$ photons must have been absorbed by an individual atom within the estimated FLASH pulse duration of 10 to $20 \mathrm{fs}$. In a first theoretical study, this high degree of photoionization was explained by a sequence of oneand multiphoton ionization processes in which an ion created in a preceding step represents the target for a subsequent step [13]. Sequential multiphoton ionization has explained fairly well recent experiments at soft or hard X-ray lasers [14-17]. In the case of $\mathrm{Xe}^{21+}$, however, this approach has to consider at least 19 steps to generate the high charge state from neutral $\mathrm{Xe}$, which is related to 19 coupled differential rate equations with 19 individual multiphoton ionization cross sections. So far, the latter could not be calculated from first principles but were only obtained through a technique of scaling, with occasional adjustment to the experimental data. An $a b$ initio calculation which describes the interaction of 60 individual photons with the 54 electrons of the Xe atom within the framework of perturbation theory seems to be, in fact, a challenging task, in particular if electron-electron correlation is taken into account. 
In the spectral range of optical radiation, photon-electron interactions at ultra-high intensities may also be described by so-called non-perturbative theories [10-12]. Their applicability depends on the ponderomotive energy $U_{p}$ :

$$
U_{p} / \mathrm{eV} \approx 1.44 \frac{I /\left(10^{13} \mathrm{~W} \mathrm{~cm}^{-2}\right)}{(\hbar \omega / \mathrm{eV})^{2}}
$$

which represents the quiver energy transferred from the oscillating field of an electromagnetic wave with frequency $\omega$ and irradiance $I$ to a free electron. To a good approximation, this expression is valid also for a weakly bound electron, e.g. in a Rydberg state, but not for a bound inner-shell electron. In the EUV and (soft) X-ray regime, however, the photoelectric effect [18], i.e. the photoexcitation of bound inner and outer electrons into the continuum is the dominant process. Weakly bound electrons and Rydberg states generally play a minor role. Thus, semi-classical theories which are based on the ponderomotive motion of quasi-free electrons are not adequate to describe strong-field phenomena in the EUV and soft X-ray range. In the present work, therefore, the idea of the individual motion of a quasi-free outer-shell electron in the field of an optical wave is extended to collective plasma oscillations of correlated bound inner-shell electrons.

\section{Outline of the model}

The idea of plasma excitations has initially been developed for the electron gas in a solid metal as described in textbooks [19]. For many years, however, models of collective electron oscillations have already been applied to describe also the so-called atomic giant resonances of $\mathrm{Xe}$ and the subsequent elements in the EUV at low photon intensities [20-23]. These strong and broad continuum resonances represent prime examples for the impact of electron correlation on inner-shell photoionization. In the following, the simplest approximations for plasma oscillations, i.e. the equations of a damped harmonic oscillator are used to express the energy transfer from a high-intensity EUV field to the Xe atom in terms of its giant resonance. For this purpose, we start with the displacement of the negative electron charge distribution of an atom from its equilibrium position as shown in figure 1. Binding to the positive nucleus induces a reset force which in first order approximation is proportional to the displacement. It results in a square binding potential and the differential equation of a damped harmonic oscillator, here excited by an oscillating force $F \cos (\omega t)$ :

$$
\ddot{x}+2 \gamma \dot{x}+\omega_{\text {res }}^{2} x=\frac{F}{N m} \cos (\omega t)
$$


Equation 2 relates to the displacement in $x$-direction of a charge distribution of $N$ electrons with the electron mass $m$ whose structure is not affected. The resonance frequency is denoted by $\omega_{\text {res }}$ and the damping ratio by $\gamma / \omega_{\text {res }}$ so that the solution of equation 2 may be expressed by:

$$
x(t)=x_{0} \cos (\omega t-\phi) \quad x_{0}=\frac{(F / N m)}{\sqrt{\left(\omega_{\text {res }}^{2}-\omega^{2}\right)^{2}+(2 \gamma \omega)^{2}}}
$$

with the phase $\phi$. In the case of an electromagnetic force with irradiance $I$ :

$$
I=\frac{c \varepsilon_{0}}{2} E^{2}=\frac{c \varepsilon_{0}}{2 e^{2}} F^{2},
$$

where $c$ denotes the speed of light, $\varepsilon_{0}$ the vacuum permittivity, $E$ the electric field strength, and $e$ the elementary charge, the kinetic energy of the electrons is given by:

$$
\begin{aligned}
& U_{k i n}=\frac{N m \dot{x}^{2}}{2}=\frac{N m x_{0}^{2} \omega^{2}}{2} \sin ^{2}(\omega t-\phi) \\
& \bar{U}_{k i n}=\frac{N m x_{0}^{2} \omega^{2}}{4}=\pi R_{y} a_{B}^{2} \alpha \hbar \frac{N I\left(\frac{2 \hbar \omega}{\hbar \omega_{\mathrm{res}}+\hbar \omega}\right)^{2}}{\left(\hbar \omega_{\mathrm{res}}-\hbar \omega\right)^{2}+(\hbar \gamma)^{2}\left(\frac{2 \hbar \omega}{\hbar \omega_{\mathrm{res}}+\hbar \omega}\right)^{2}} .
\end{aligned}
$$

Here, $a_{B}$ denotes the Bohr radius, $R_{y}$ the Rydberg energy, and $\alpha$ the fine structure constant. For a single free electron without any binding, one obtains the kinetic energy from equation $5 \mathrm{~b}$ by setting $N=1, \omega_{\text {res }}=0$ and $\gamma=0$ which leads directly to equation 1 . Hence, the present model so far represents also an extension for the classical treatment of ponderomotive motion from a single free electron to many correlated electrons which are bond.

In the presence of a binding $\left(\omega_{\text {res }} \neq 0\right)$, the potential energy is given by:

$$
\begin{aligned}
& U_{p o t}=\frac{N m \omega_{\mathrm{res}}^{2} x^{2}}{2}=\frac{N m x_{0}^{2} \omega_{\mathrm{res}}^{2}}{2} \cos ^{2}(\omega t-\phi) \\
& \bar{U}_{p o t}=\frac{N m x_{0}^{2} \omega_{\mathrm{res}}^{2}}{4}=\frac{\omega_{\mathrm{res}}^{2}}{\omega^{2}} \bar{U}_{k i n} .
\end{aligned}
$$

Moreover, for a damped oscillation $\left(\omega_{\text {res }} \neq 0, \gamma \neq 0\right)$, the damping energy may be calculated according to: 


$$
\begin{aligned}
& U_{\text {damp }}=\int 2 N m \gamma \dot{x} d x=\int_{0}^{t} 2 N m \gamma \dot{x}^{2} d t^{\prime}=2 N m \gamma x_{0}^{2} \omega^{2} \int_{0}^{t} \sin ^{2}(\omega t-\phi) d t^{\prime} \\
& \bar{U}_{\text {damp }}=\gamma t N m x_{0}^{2} \omega^{2}=4 \gamma t \bar{U}_{k i n} .
\end{aligned}
$$

According to equation $7 \mathrm{~b}$, the mean damping energy absorbed by the system increases linearly with time, whereas the averaged kinetic and potential energies, according to equations $5 \mathrm{~b}$ and $6 \mathrm{~b}$, remain constant in the case of a stationary oscillation as discussed here. For the total energy of the oscillation, one obtains therefore:

$$
\begin{gathered}
\bar{U}_{t o t}=\bar{U}_{k i n}+\bar{U}_{p o t}+\bar{U}_{d a m p}=\bar{U}_{k i n}\left(1+\frac{\omega_{\mathrm{res}}^{2}}{\omega^{2}}+4 \gamma t\right) \\
\dot{\bar{U}}_{t o t}=\dot{\bar{U}}_{d a m p}=4 \gamma \bar{U}_{k i n}=4 \gamma \bar{U}_{t o t}\left(1+\frac{\omega_{\mathrm{res}}^{2}}{\omega^{2}}+4 \gamma t\right)^{-1} \\
=4 \pi R_{y} a_{B}^{2} \alpha \frac{N I \hbar \gamma\left(\frac{2 \hbar \omega}{\hbar \omega_{\mathrm{res}}} \hbar \hbar\right)^{2}}{\left(\hbar \omega_{\mathrm{res}}-\hbar \omega\right)^{2}+(\hbar \gamma)^{2}\left(\frac{2 \hbar \omega}{\hbar \omega_{\mathrm{res}}+\hbar \omega}\right)^{2}} .
\end{gathered}
$$

Finally, the total energy of our damped plasma oscillation is related to the cross section of the corresponding photoexcitation process which is defined as the rate of absorbed photons per atom $\dot{N}_{a b s} / N_{a t}$ normalized to the photon flux density $I / \hbar \omega$ :

$$
\sigma=\frac{\dot{N}_{a b s} / N_{a t}}{I / \hbar \omega}=\frac{\dot{\bar{U}}_{t o t}}{I}=4 \pi R_{y} a_{B}^{2} \alpha \frac{N \hbar \gamma\left(\frac{2 \hbar \omega}{\hbar \omega_{\mathrm{res}}+\hbar \omega}\right)^{2}}{\left(\hbar \omega_{\mathrm{res}}-\hbar \omega\right)^{2}+(\hbar \gamma)^{2}\left(\frac{2 \hbar \omega}{\hbar \omega_{\mathrm{res}}+\hbar \omega}\right)^{2}}
$$

\section{Application of the model}

Equation 9 may now be applied to fit the data points of the photoabsorption cross section of Xe in the range of the $4 d$ giant resonance, as shown by the thick solid line in figure 2 . The agreement between the model curve and the experimental data is fairly well. As a result, one obtains within $1 \%$ standard uncertainty the fitting parameters $\hbar \omega_{\text {res }}=100.0 \mathrm{eV}, \hbar \gamma=17.2 \mathrm{eV}$, and $N=14$. The latter value is higher than the oscillator strength of the ten $4 d$ electrons only but lower than the oscillator strength of the eighteen $4 d, 5 p$, and $5 s$ electrons which are known to all couple to the $4 d$ giant resonance [20-23]. 
In a further step, one may calculate now the total energy of the oscillating $4 d, 5 p$, and $5 s$ shells in Xe according to the equations $8 \mathrm{a}$ and $5 \mathrm{~b}$ and obtains in the resonance case $\left(\hbar \omega=\hbar \omega_{\mathrm{res}}\right)$ :

$$
\bar{U}_{t o t} / \mathrm{eV} \approx 1.79 \times\left(I / 10^{13} \mathrm{~W} \mathrm{~cm}^{-2}\right) \times(t / \mathrm{fs}) .
$$

Equation 10 is valid for $t>(2 \gamma)^{-1} \approx 0.02 \mathrm{fs}$, e.g. for sufficiently long photon pulses as in the case for FLASH with a pulse duration of /10 fs at about $100 \mathrm{eV}$ photon energy [1]. At FLASH, furthermore, the basic assumption for the present model of a stationary oscillation is valid because the FLASH pulse duration is also more than two orders of magnitude longer than the oscillation period of $2 \pi / \omega_{\text {res }}=$ $h / 100 \mathrm{eV} \approx 0.04$ fs.

The linear temporal increase of the total energy with time in equation 10 is due to the accumulation of damping energy within the system as already expressed by equation 7b. Apart from radiative and nonradiative emissions, internal friction within the plasma due to electron-electron correlation and coupling may, cause damping, in particular if the damping is as high as for the very broad $4 d$ giant resonance in Xe with $\gamma / \omega_{\text {res }}=0.172$. The accumulation of damping energy by the atom leads, then, to the heating of its electron system which, thus, becomes a sort of hot sub-nano electron plasma from which, statistically, electrons may gain sufficient energy to leave the atom. The mean FLASH pulse duration scales with the wavelength and may meanwhile be estimated to amount to $t=(15 \pm 5)$ fs in the EUV at about $13 \mathrm{~nm}$ for the period from 2006 to 2009 when the Xe results [8,9] were obtained. It is, thus, about $50 \%$ longer than previously assumed [24]. With this value for $t$, one may, hence, according to equation 10, calculate the absorbed plasma heating energy of the $4 d$ (and $5 p$ and $5 s$ ) electrons in $\mathrm{Xe}$ as a function of irradiance $I$ and from that, using literature data for the respective ionization potentials [25], the highest Xe charge state which may be reached at FLASH at about 100 $\mathrm{eV}$ photon energy as a function of irradiance. The result is shown by the rectangles in figure 3 in comparison to the corresponding experimental data obtained at 90.5 and $93 \mathrm{eV}$ photon energy [8,9], which are shown by the filled circles.

Since the total energy absorbed by an atom may be partially transferred also as excess energy to the emitted photoelectrons and - as a result of subsequent non-radiative decay of inner-shell vacancies - to emitted Auger electrons, the measured highest charges must be lower than those calculated from the total energy absorbed and the respective ionization potentials. This is the case for all irradiances except for the lowest at $1.4 \times 10^{14} \mathrm{~W} \mathrm{~cm}^{-2}$, as can be seen in figure 3. However, this point is already close to the lower limit for the classical calculation of energy absorbed per atom from a radiation field which, since Einstein [18], has been defined by: 


$$
\bar{U}_{t o t} \gg \hbar \omega
$$

and for the present case, according to equation 10 with $t=15 \mathrm{fs}$, by $I \gg 4 \times 10^{13} \mathrm{~W} \mathrm{~cm}^{-2}$. For lower irradiances, the distribution of energy among the target atoms is no longer homogeneous but rather statistical and those atoms which are hit by photons absorb more than the mean absorption energy per atom. Accordingly, the achievable charge states here should be, in fact, generally higher than predicted by the present model. It should be noted that the limit of the present semi-classical approach as defined by equation 11 corresponds also to the limit for ponderomotive energy of (quasi-)free electrons to, again, distinguish the non-perturbative from the perturbative regime [10-12].

At high irradiances, the measured highest charge states of Xe shown in figure 3 seem to be limited to about 22 (dashed line in figure 3), which is not the case for the calculated values. The latter just increase monotonically with the mean energy absorbed per atom. In practice, however, the highest charge state is limited by the number of electrons which may be involved, either in the primary photoionization process or in the subsequent Auger decay. In the present case of the $4 d$ giant resonance in $\mathrm{Xe}$, these are the ten $4 d$ electrons and the eight $5 s$ and $5 p$ electrons, i.e. 18 , which might be slightly further increased by $4 p-4 d$ mixing [26].

\section{Conclusion}

In conclusion, the present semi-classical model represents a straightforward extension of theories for ponderomotive motion of outer quasi-free electrons in strong laser fields to strongly correlated innershell electrons in the approximation of a harmonic potential for binding. In the resulting solution of a damped harmonic plasma oscillation, damping is interpreted to be mainly due to electron-electron correlation. In this view, the damping energy absorbed from the field leads to a sort of electron heating and, consequently, to electron emission. Fair agreement with experimental data is obtained for the total energy absorbed and the highest charge state achievable in the case of Xe excited in the extreme ultraviolet at ultra-high intensities. The model may be used as a starting point to calculate from the energy transfer to the atom also the intensity dependences of the individual charge states by means of a suitable statistical model in analogy to recent cluster theories [27].

\section{Acknowledgement}

The author would like to thank S. V. Bobashev, A. Kato, B. F. Sonntag, A. A. Sorokin, K. Tiedtke, G. Ulm, and P. Zimmermann for support und many helpful discussions. 


\section{References}

[1] Ackermann W et al 2007 Nat. Photonics 1336

[2] Shintake T et al 2008 Nat. Photonics 2555

[3] Emma P et al 2010 Nat. Photonics 4641

[4] O'Shea P G and Freund H P 2001 Science 2921583

[5] Kapteyn H C and Ditmire T 2002 Nature 420467

[6] Feldhaus J, Arthur J and Hastings J B 2005 J. Phys. B: At. Mol. Opt. Phys. 38 S799

[7] Bostedt C et al 2009 Kai Siegbahn Memorial Volume of Nucl. Instr. and Meth. A 601108

[8] Sorokin A A et al 2009 Phys. Rev. Lett. 99213002

[9] Richter M et al 2009 Phys. Rev. Lett. 102163002

[10] Keldysh L V 1965 Sov. Phys. JETP 201307

[11] Protopapas M, Keitel C H and Knight P L 1997 Rep. Prog. Phys. 60389

[12] Delone N and Krainov V 2000 Multiphoton Processes in Atoms (New York: Springer)

[13] Makris M G, Lambropoulos P and Mihelič A 2009 Phys. Rev. Lett. 102033002

[14] Motomura K et al 2009 J. Phys. B: At. Mol. Opt. Phys. B 42221003

[15] Rudenko A et al 2010 J. Phys. B: At. Mol. Opt. Phys. B 43194004

[16] Richter M et al 2010 J. Phys. B: At. Mol. Opt. Phys. B 43194005

[17] Young L et al 2010 Nature 46656

[18] Einstein A 1905 Ann. Phys. (Leipzig) 322132

[19] Kittel Ch 1976 Introduction to Solid State Physics (New York: John Wiley \& Sons)

[20] Fano U and Cooper J W 1968 Rev. Mod. Phys. 40441

[21] Parpia F A, Johnson W R and Radojević V 1984 Phys. Rev. A 293173

[22] Connerade J P, Esteva J M and Karnatak R C (eds.) 1987 Giant Resonances in Atoms, Molecules, and Solids (New York: Plenum Press) and references therein

[23] Amusia M Ya and Connerade J P 2000 Rep. Prog. Phys. 6341

[24] Mitzner R et al 2009 Phys. Rev. A 80025402

[25] Yamakawa K et al 2004 Phys. Rev. Lett. 92123001

[26] Wendin G 1981 Breakdown of the One-Electron Pictures in Photoelectron Spectra, Structure and Bonding Vol. 45 (Heidelberg: Springer)

[27] Bostedt C et al 2010 New J. Phys. 12083004 


\section{Figure captions}

Figure 1. Negative electron charge distribution of an atom (blue) oscillating in $x$-direction with respect to the positive nucleus (red).

Figure 2. Photoabsorption cross section of $\mathrm{Xe}$ at the $4 d$ giant resonance (experimental data: points; Relativistic Time-Dependent Local Density Approximation (RTDLDA): thin solid line; Relativistic Random Phase Approximation (RRPA): dashed line) [21]. The thick solid line shows the fitting result of the present model (equation 9) with $\hbar \omega_{\text {res }}=100.0 \mathrm{eV}, \hbar \gamma=17.2 \mathrm{eV}$, and $N=14 . \omega=1$ a.u. corresponds to $\hbar \omega \approx 27.2 \mathrm{eV}, 1 \mathrm{Mb}=$ $10^{-18} \mathrm{~cm}^{2}$.

Figure 3. Highest charge states of Xe generated by $(15 \pm 5)$ fs EUV pulses as a function of irradiance (filled circles: experimental data $[8,9]$; rectangles: calculated within the present model for the pulse duration range from 10 to $20 \mathrm{fs})$. 


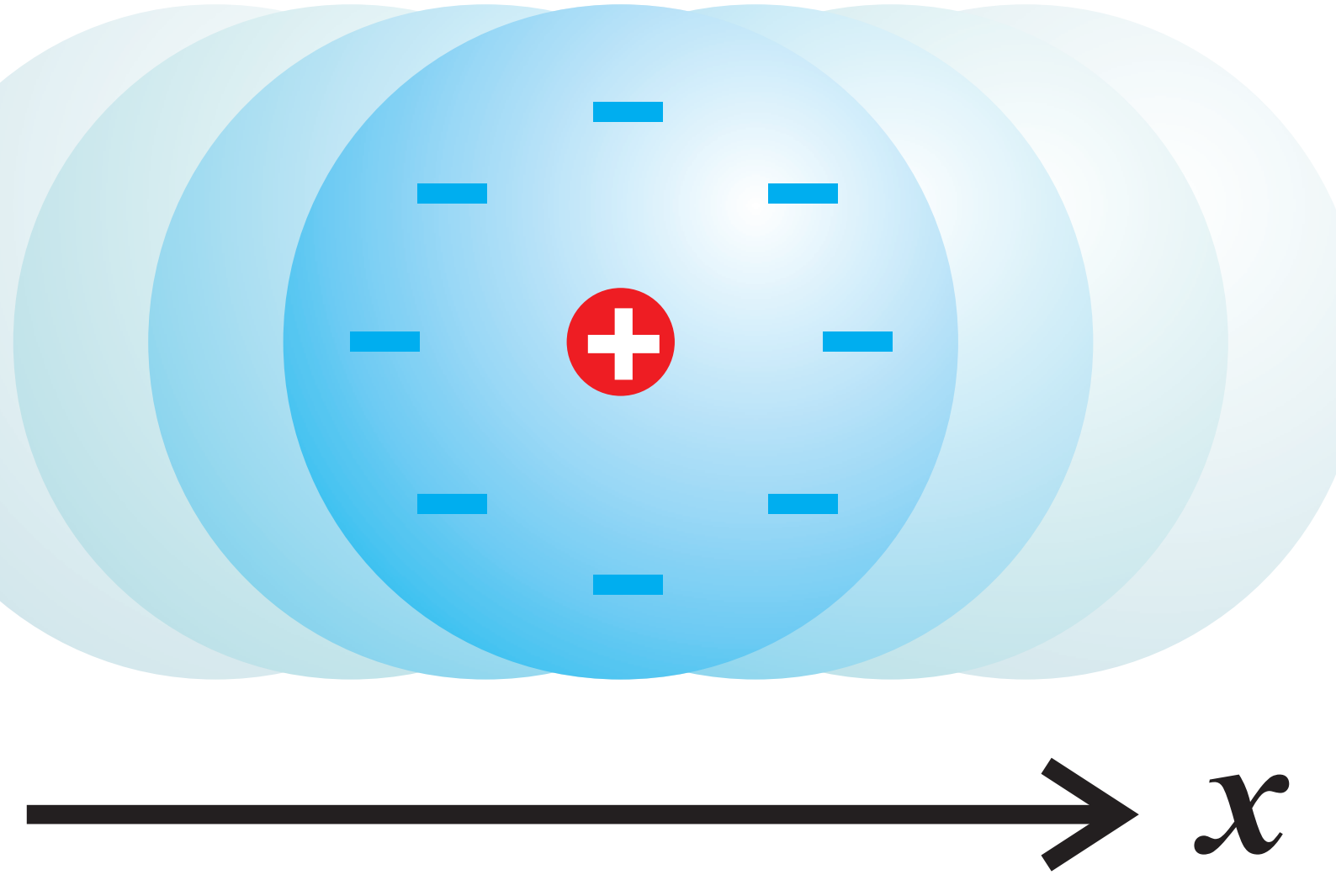

Figure 1 (Fig1_(new)Displacement.eps) 


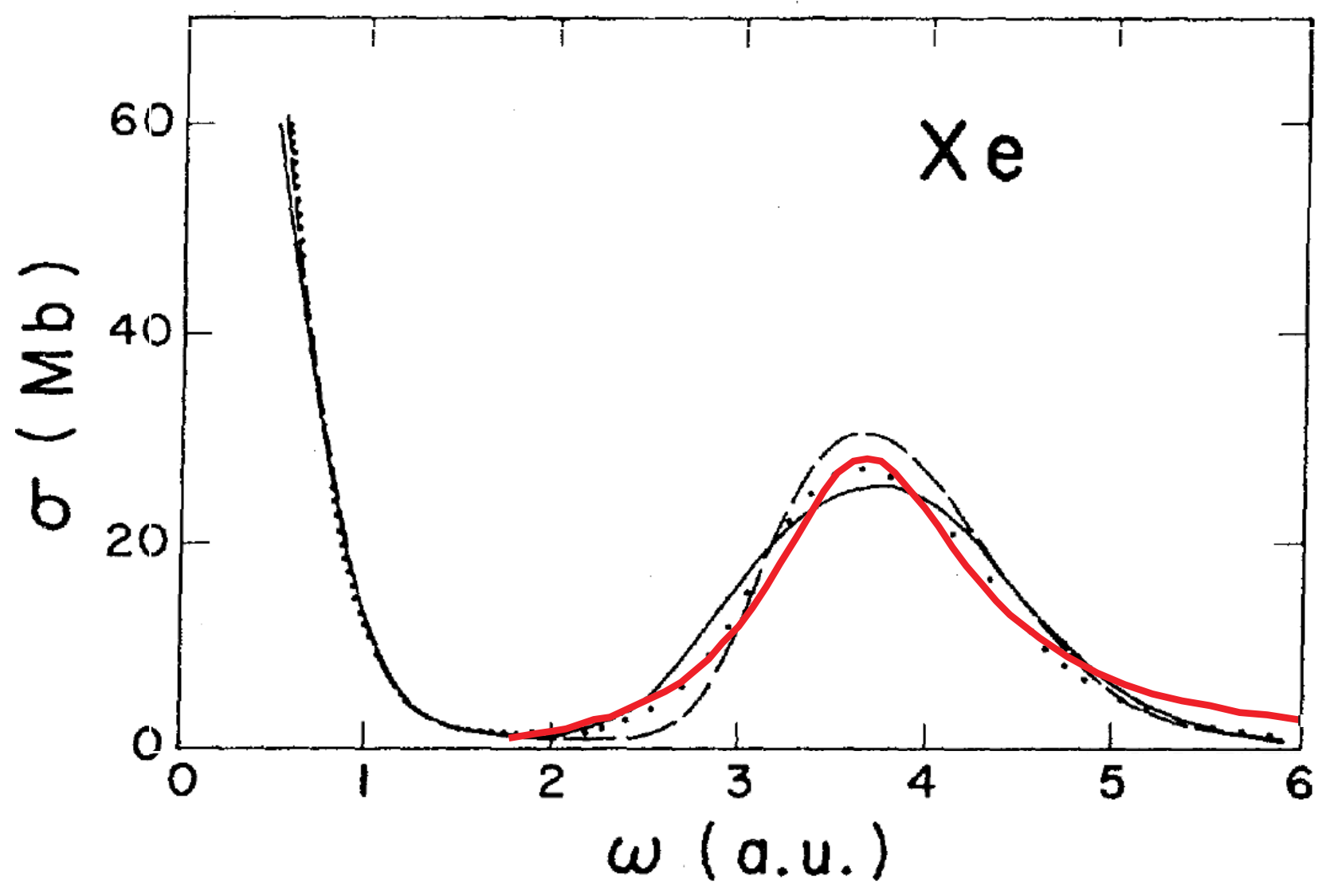

Figure 2 (Fig2_Xe4d.eps) 


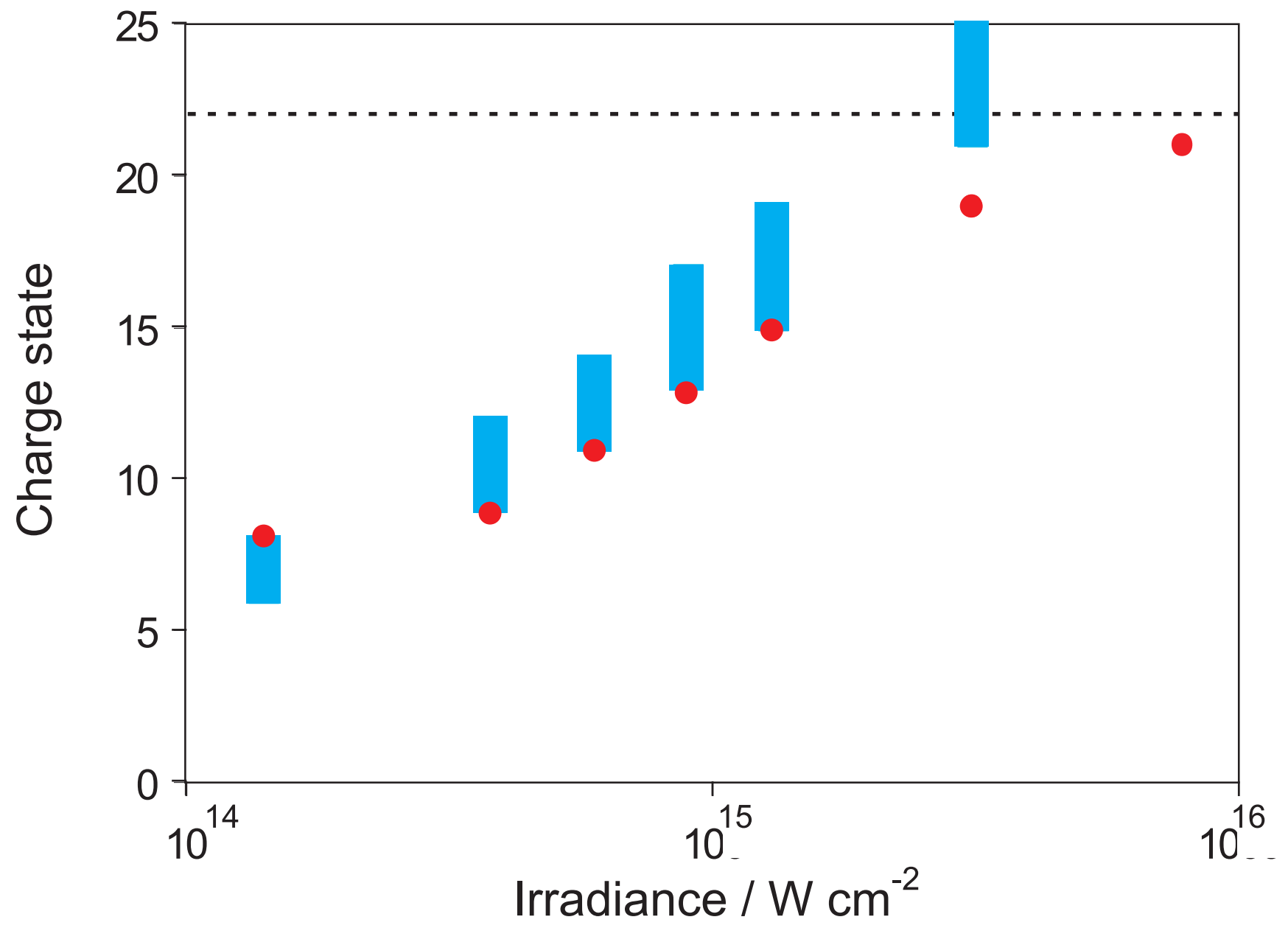

Figure 3 (Fig3_Xe4ChargeStates_vs3.eps) 